\title{
Balkanologie
}

Balkanologie Revue d'études pluridisciplinaires

Vol. XIV, $\mathrm{n}^{\circ}$ 1-2 | 2012

Volume XIV Numéro 1-2

\section{Gerald W. Creed, Masquerade and Postsocialism. Ritual and Cultural Dispossession in Bulgaria}

Bloomington and Indianapolis: Indiana University Press, 2011, 254 pages

Katerina Seraïdari

\section{OpenEdition}

\section{Journals}

Édition électronique

URL : http://journals.openedition.org/balkanologie/2356

DOI : 10.4000/balkanologie.2356

ISSN : 1965-0582

Éditeur

Association française d'études sur les Balkans (Afebalk)

Référence électronique

Katerina Seraïdari, « Gerald W. Creed, Masquerade and Postsocialism. Ritual and Cultural Dispossession in Bulgaria », Balkanologie [En ligne], Vol. XIV, n 1-2 | 2012, mis en ligne le 17 janvier 2013, consulté le 17 décembre 2020. URL : http://journals.openedition.org/balkanologie/2356 ; DOI : https://doi.org/ 10.4000/balkanologie.2356

Ce document a été généré automatiquement le 17 décembre 2020.

(c) Tous droits réservés 


\title{
Gerald W. Creed, Masquerade and Postsocialism. Ritual and Cultural Dispossession in Bulgaria
}

Bloomington and Indianapolis: Indiana University Press, 2011, 254 pages

\author{
Katerina Seraïdari
}

\section{RÉFÉRENCE}

Gerald W. Creed, Masquerade and postsocialism. Ritual and Cultural Dispossession in Bulgaria, Bloomington and Indianapolis : Indiana University Press, 2011, 254 p.

Je remercie Ilia Iliev d'avoir lu et commenté une version précédente de ce compte-rendu.

Basée sur des enquêtes de terrain dans quarante-quatre villages et quatre villes, cette étude fascinante examine un rituel bulgare, appelé kukeri ou survakari. Celui à qui sont familiers les rituels religieux (mais aussi carnavalesques) en Grèce aura tout d'abord l'impression d'une grande similitude entre les cérémonies dans les deux pays. Les questions de la fonction métaphysique du rituel et de sa mise en cause, de la fierté des participants envers leur village et de la rhétorique de résistance qui traverse ce type de manifestations, du rôle des migrants et de l'introduction d'innovations, de la répartition du rituel entre moments formels et informels, de la présence d'invités d'honneur et d'éléments de compétition, de la transformation des pratiques et de leur association tant à un passé immémorial qu'à la modernité, sont connues de tous ceux qui ont entrepris des recherches sur des terrains similaires dans les Balkans. Mais Creed ne replace pas ces pratiques dans un contexte régional: le fait que certains termes soient communs en Bulgarie et en Grèce (comme horo, Arapi, merak) ne retient pas son attention. Les Balkans, en tant qu'échelle d'observation et d'analyse, ne sont mentionnés qu'occasionnellement, pour souligner soit l'exceptionnalisme bulgare et une de ses facettes - le «nationalisme faible » (p. 26), soit l'existence d'un idéal de la masculinité dans les représentations et la tradition folklorique (p. 76), soit encore 
l'importance des rituels funéraires pour consolider une communauté (p. 148). Le mot "Balkans » n'apparaît même pas dans l'Index ; en revanche, le terme " Eastern Europe " est bien là, constituant l'unité géographique de référence.

Ce choix est explicité dans le titre de l'ouvrage : le but de l'auteur est d'analyser le contexte postsocialiste afin de montrer l'existence d'alternatives (voire de «modernités alternatives»), qui ont été délaissées par l'imposition du projet néolibéral. Le résultat de cet effacement de l'échelle régionale est une utilisation minimale des travaux anthropologiques sur la Grèce en faveur de travaux sur des pays comme la Pologne ou la Hongrie, censés illustrer le contexte socialiste et postsocialiste. En fait, Creed présente son livre comme «l'extension postsocialiste » de l'ouvrage de Gail Kligman (1988) sur les rituels du cycle de vie pendant la période socialiste (p. 13).

3 Le premier chapitre examine les modalités du rituel, ainsi que l'organisation des festivals qui ont été inaugurés dans les années 1960 par les responsables du Parti communiste afin de redéfinir une pratique idéologiquement suspecte (considérée comme superstitieuse) et peut-être même politiquement dangereuse, à cause de la présence de grands groupes d'hommes masqués (p. 56). Creed montre de manière subtile comment les festivités dans les villages et l'organisation des festivals sont aujourd'hui interdépendantes. Il étudie avec une attention particulière les facteurs économiques, ce qui le conduit à utiliser un champ lexical économique lui aussi pour décrire les évolutions festives, comme, par exemple, quand il analyse la tendance à fabriquer et à utiliser des masques de plus en plus grands: il parle de «mask inflation» (p. 66).

4 Le deuxième chapitre s'intéresse au genre et à la sexualité. Après un état des lieux sur la manière dont l'autorité masculine a été mise en question pendant les périodes socialiste et postsocialiste, l'auteur examine comment ce rituel permet aux hommes de s'ériger en défenseurs de la nation au travers du maintien de pratiques centrales pour l'identité nationale bulgare (p. 83). La participation croissante des femmes et des enfants est une innovation qui redéfinit ces rituels en tant qu'expressions de la modernité, et non comme des survivances patriarcales (p.91). Une autre échelle d'observation et d'analyse, aussi centrale que le postsocialisme pour Creed, émerge alors : l'approche globale, qui prend ici la forme d'une "global gay culture » et d'un "global sexscape ». Si sous un régime socialiste répressif ce rituel offrait un espace de liberté pour l'expression discrète du désir homosexuel, le contexte postsocialiste, qui refuse toute ambigüité dans les expressions sexuelles et qui caractérise chaque action soit comme «straight » soit comme «gay», rend certains gestes suspects et transforme les festivités en manifestation d'une homophobie partagée: «Les interprétations homophobes sont le produit d'une exposition croissante à des possibilités homosexuelles globales dans un contexte où la sécurité masculine d'antan est mise en danger et menacée» (p.99). Avec une grande finesse, Creed montre comment l'individualisme libéral impose l'idée que les hommes sont responsables de leur identité sexuelle (p. 101). Mais la présence de modèles globaux qui introduisent une nouvelle compréhension essentialiste de la sexualité (selon le schéma binaire homo/hétéro, qui semble remplacer les catégories du passé centrées sur une perception fixe de la masculinité et de la féminité) finit par intensifier l'homophobie et révèle l'insuffisance des discours sur les Droits de l'Homme.

Le troisième chapitre examine le concept de "société civile " et pose la question de savoir dans quelle mesure certains éléments habituellement associés avec la société 
civile peuvent se retrouver dans ces rituels. Les participants s'organisent en groupes, comme s'il s'agissait d'un «club informel » (p. 120) ou d'une "fraternité à l'échelle nationale avec des branches locales» (p. 123). Comme l'auteur le dit un peu plus bas, «Ceci n'est pas une analyse sophistiquée, et c'est justement là où je veux en venir. Il ne faut pas être un génie pour voir les caractéristiques de la société civile dans ces activités, et pourtant elles ne sont jamais incluses dans ces discussions et ces analyses » (p. 128). La conclusion de ce chapitre est que le concept de société civile ne prend pas en compte ce qui est informel ou qui n'a pas une structure permanente. Par la suite, Creed n'hésite pas à caractériser la participation à ce rituel comme étant de l'activisme (voir par exemple p. 157). Creed aurait pu s'inspirer des travaux sur la Grèce comme celui de Dimitri A. Sotiropoulos ${ }^{1}$, qui évoque l'existence d'une "société civile informelle » et parallèle, définie, entre autres, par des festivités et des fêtes régulières qui ont lieu à Pâques ou durant l'été.

6 Le quatrième chapitre examine la relation entre l'espace domestique, la famille, le village et la communauté, tandis que le chapitre suivant traite la question de l'ethnicité et de la participation des Roms. L'analyse de Creed suggère que les Roms ne reconnaissent pas ces rituels comme un lieu de revendication ou de discrimination. Le discours global joue aussi un rôle : les jeunes Roms valorisent les produits culturels associés aux Afro-américains et les Roms de tout âge idolâtrent Martin Luther King (p.176). L'Union Européenne, en tant qu'acteur politique, apparaît également: le respect des droits des minorités était une condition nécessaire afin que la Bulgarie puisse en devenir membre (p. 193). L'idée centrale de ce chapitre est que les « relations d'inclusion ambivalente ", qui sont le fruit d'expériences locales, ont été remplacées par une idée générique de tolérance et de multiculturalisme, produite par le libéralisme occidental.

7 Si la particularité de ces alternatives locales (une perception différente de la sexualité, une participation civique au travers des rituels, une «inclusion ambivalente» de l'Autre) pose problème à toute forme de projet étatique, c'est parce qu'il faut des solutions uniformes à des problèmes génériques. La disparition de ces alternatives locales conduit à la dépossession culturelle et rituelle, idée centrale que l'on retrouve déjà dans le titre de l'ouvrage et qui rappelle le principe marxiste d'aliénation. Le titre de la conclusion est aussi particulièrement bien choisi: "Modernity in drag", « Modernité travestie », ce qui permet à l'auteur d'être optimiste, dans la mesure où le travestissement a un potentiel disruptif (p. 217).

8 Avec beaucoup de sensibilité et de maîtrise, Creed réussit à redéfinir l'importance de l'étude des rituels - jusqu'ici facilement expédiés en tant que formes folkloriques. Mais si «le postsocialisme est une condition globale» (p.5), si l'imposition des valeurs néolibérales ne concerne pas seulement les pays ex-communistes, comment définir la spécificité du domaine des "études postsocialistes"? Si Creed se situe dans ce domaine, il ne s'agit pas d'un choix géographique (l'Europe de l'Est vs les Balkans), mais d'un choix théorique et d'orientation des débats. Il est pourtant temps d'interroger la pertinence de ce domaine d'études qui fonde sa légitimité sur l'étude d'un projet étatique (le socialisme) ayant eu une durée de vie très limitée. Si les trajectoires des pays postsocialistes sont aujourd'hui très variées et si des discours globaux exercent une influence écrasante, en quoi ce domaine d'étude continue-t-il de structurer un espace délimité ? L'effort de Michael Herzfeld ${ }^{2}$ d'établir un cadre comparatif entre la Grèce et la Thailande, en s'appuyant sur le concept de "crypto-colonialisme », est 
caractéristique de la nécessité de se référer à un domaine d'étude bien établi dans le monde académique afin de légitimer une approche inhabituelle. Autrement dit, il semble être plus facile de comparer la Grèce et la Thaïlande dans la mesure où le concept de " crypto-colonialisme » permet à la comparaison d'entrer dans le cadre des études postcolonialistes, que de comparer la Grèce et la Bulgarie (puisque la deuxième constitue un objet d'études postsocialistes contrairement à la première).

Le lecteur pourrait avoir le sentiment que Creed s'intéresse moins aux données locales marquées par des interactions de longue date avec des voisins qui, certes, n'ont pas connu le régime socialiste mais qui ont subi les mêmes formes de dépossession à cause du néolibéralisme. Ainsi, la crise grecque qui, selon une partie de la population, tend à transformer le pays « en Bulgarie ", nous oblige peut-être à reconsidérer la spécificité du monde postsocialiste. Si Creed parvient à contester la logique des catégorisations thématiques (c'est-à-dire la distinction entre anthropologie politique, religieuse et économique, puisque ces domaines sont examinés ensemble dans son livre), s'il arrive à dépasser la logique des « aires culturelles » (Balkans, Méditerranée), il aurait également pu interroger la pertinence du clivage que les « études postsocialistes » ont introduit. Cela dit, il s'agit indiscutablement d'un livre remarquable qui pousse à la réflexion.

\section{NOTES}

1. Sotiropoulos (Dimitri A.), «Formal weakness and informal strength: Civil society in Contemporary Greece », Discussion Paper (16), The Hellenic Observatory, February 2004, p. 14.

2. Herzfeld (Michael), "The absent presence. Discourses of crypto-Colonialism», The South Atlantic Quarterly, 101 (4), 2002. 